\title{
Morphologic changes in the microcirculation induced by chronic smoking habit: A videocapillaroscopic study on the human labial mucosa
}

Raffaele Molino Lova, MD, ${ }^{a}$ Benedetta Miniati, MD, ${ }^{a}$ Claudio Macchi, MD, ${ }^{\text {a }}$ Massimo Gulisano, MD, ${ }^{b}$ Gherardo Gheri, MD, ${ }^{b}$ Claudio Catini, MD, ${ }^{b}$ Andrea A. Conti, MD, ${ }^{\text {a,c }}$ and Gian Franco Gensini, MD ${ }^{a, c}$ Florence, Italy

Background Cigarette smoking has been identified as a major risk factor for cardiovascular and pulmonary diseases. Although an impressive literature does exist on the subject, no evidence is available on morphologic changes induced with chronic smoking habit in the human microcirculation.

Subjects and Methods One hundred healthy subjects, 50 smokers and 50 nonsmokers, underwent videocapillaroscopy of the labial mucosa. For each subject, the caliber of capillary loops, the number of visible capillary loops, the background optical transmittance, the tortuousness of capillary loops, the presence of microaneurysms, the presence of microhemorrhages, and the cumulative smoking habit (pack-year index) were noted.

Results Smokers had a lower caliber of capillary loops $(P<.001)$, with a higher number of visible capillary loops $(P$ $<.001)$, a lower background optical transmittance $(P<.001)$, and a more marked tortuousness of capillary loops $(P<.001)$. Microaneurysms and microhemorrhages were absent in nonsmokers, and 1 smoker of 3 had microaneurysms alone, and 1 smoker of 3 had both microaneurysms and microhemorrhages. A significant correlation was found between cumulative smoking habit and tortuousness of capillary loops $(P<.001)$ and between cumulative smoking habit and total score (tortuousness score + microaneurysm score + microhemorrhage score; $P<.005)$.

Conclusion Chronic smoking habit does induce significant morphologic changes in the microcirculation of the human labial mucosa, and these changes can be easily and comfortably recorded with videocapillaroscopy. (Am Heart J 2002; 143:e2.)

Cigarette smoking has been identified as a major risk factor for cardiovascular and pulmonary diseases, and an impressive literature does exist on the subject. As regards the microcirculation, although valuable papers have been published about the hemodynamic and pharmacologic effects of smoke,,$^{1,2}$ about smoke-induced leukocyte-endothelium interactions, ${ }^{3}$ and about the effects of smoke on leukocytes deformability ${ }^{4}$ and blood rheology, 5 no evidence is available on morphologic changes in the human microcirculation induced with chronic smoking habit.

The aim of this paper was to investigate these changes with videocapillaroscopy. This recent technique shows

From the aDepartment of Cardiovascular Medicine, Don Gnocchi Foundation, and the b/nstitute of Anatomy, and the 'Department of Internal Medicine and Cardiology, University of Florence.

Submitted January 19, 2001; accepted April 26, 2001

Reprint requests: Raffaele Molino Lova, MD, Via Mannelli, 7, 50136 Florence,

Italy.

E-mail: raffmoli@tin.it

Copyright 2002, Mosby, Inc. All rights reserved.

1097-6744/2002/\$35.00+0 4/90/121461

doi: $10.1067 / \mathrm{mhj} .2002 .121461$ many advantages with respect to the traditional capillaroscopy. In fact, videocapillaroscopy is a simple, safe, sensitive, reliable, and low-cost technique, comfortable for the patient and easy to perform for the operator. Furthermore, it can be performed, by bedside, on any part of the skin and of clinically accessible mucosae. The choice to perform videocapillaroscopy on the labial mucosa was related to the accessibility and comfort of the investigation, to the good quality of the high magnification-obtained pictures, and to the possibility of investigation of the effects of smoke on the microcirculation of a mucosa directly in contact with it.

\section{Subjects and Methods}

One hundred healthy subjects, 50 smokers and 50 nonsmokers, underwent labial mucosa videocapillaroscopy in our laboratory. All the subjects gave their written informed consent for processing and use of personal medical data in scientific papers, in accordance to the Italian Law. The study was approved by the Ethical Committee of the Don Gnocchi Foundation.

Both smokers and nonsmokers groups were comprised of 25 
men and 25 women, and the ages ranged from 24 to 56 years (mean \pm standard deviation [SD], $43 \pm 8.5$ years) in the smokers group and from 26 to 59 years (mean \pm SD, $44 \pm 8$ years) in the nonsmokers group ( $P=$ not significant). In the smokers group, the habit lasted for at least 10 years and the consumption of filter cigarettes ranged from 10 to 30 cigarettes per day. The cumulative effect of smoke, measured with the "pack-year" index (number of cigarette packages smoked every day times the years of smoking habit), ranged from 5 to 52 (mean $\pm \mathrm{SD}, 24.5 \pm 12$ ). Alcohol and coffee consumption was normal for all the subjects, and no subject had a drug dependency (opiates, cocaine). Clinical examination, instrumental diagnostic, and laboratory test results excluded, for all the subjects, any known systemic disease capable of affecting microcirculation. All fertile female subjects were examined between the 7 th and the 10th day after menstruation, and none were taking oral contraceptives. A preliminary dentistry consultation excluded any mouth disease. Cardiac and pulmonary function test results, including arterial blood gases, were normal in all the subjects.

Videocapillaroscopy was performed by the same skilled operator, at the same room temperature $\left(20^{\circ} \mathrm{C}\right)$, with the subject in the supine position, in all cases in the morning, at least 2 hours after the breakfast, and, for the smokers group, at least 2 hours from the last smoked cigarette. Videocapillaroscopy was performed with a Videocap apparatus from Scalar Co Ltd (Tokyo Japan), controlled by the Videocap 5.1 Plus software, from the same manufacturer, equipped with a contact optical probe $500 \times$ magnification, corresponding to a field of $0.25 \mathrm{~mm}^{2}$. Although we a priori assumed a limited intrasubject variability, 5 pictures were taken for each subject, dividing the mucosa of the slightly extroverted lower lip into 5 ideal sectors, halfway between the basis of the gum and the border of the mucosa of the lip.

All the pictures were examined by 2 independent observers, blinded as to whether the subject was a smoker or a nonsmoker. The intraobserver and interobserver variability was assessed with the 2 observers evaluating twice the same randomly selected 100 pictures. Two sets of data, parametric and nonparametric, were collected.

\section{Set A: Parametric data}

The parametric data included the following data: 1, caliber of visible capillary loops (average value obtained from 3 measurements for each capillary loop); 2 , number of visible capillary loops in each picture; 3 , background optical transmittance (an expression of the circulatory state of precapillary arteriolae and postcapillary venulae, located in deeper planes and hence not visible) measured with the Image-Pro Plus 4.0 software, from Media Cybernetics (Silver Spring, Md), after conversion of the original color picture in a 16-bit grayscale picture, calibrated at 0 for the black and at 256 for the white, positioning the sample in the intercapillary space. Ten readings were made for each picture and then averaged. For all the set A data, the obtained values in any single picture were averaged among the 5 pictures of each subject.

\section{Set B: Nonparametric data}

The nonparametric data included the following data: 1, tortuousness of capillary loops, scored from 0 to 3 , on a morphologic basis ( 0 , no tortuousness or "hairpin" aspect; 1 , single twist tortuousness; 2 , multiple twist tortuousness or "corkscrew" aspect; 3, complete distortion of the capillary loop or "ball" aspect; Figures 1 and 2); 2, microaneurysms, scored 0 or 1 , on a categoric basis ( 0 , absence of microaneurysms; 1 , presence of microaneurysms; Figure 3); 3, microhemorrhages, scored 0 or 1 , on a categoric basis $(0$, absence of microhemorrhages, 1, presence of microhemorrhages; Figure 3). The total score was obtained with simply summing up the tortuousness score, the microaneurysm score, and the microhemorrhage score. For the set B data, the highest score found in any of the 5 pictures was considered the final score of the subject.

The statistical significance of the differences between the smokers and the nonsmokers groups was checked with the Student $t$ test for independent samples, with regard to parametric data, and with the Mann-Whitney rank test, with regard to nonparametric data. The hypothesis of a linear correlation between the parametric data and cumulative smoking habit was tested with the Pearson product moment correlation coefficient, and the hypothesis of a correlation between the nonparametric data and cumulative smoking habit was tested with the Spearman rank correlation coefficient.

\section{Results}

Intrasubject variability satisfied the a priori hypothesis of a limited dispersion. For the 3 parametric data, variability ranged between $+5 \%$ and $-5 \%$ with respect to the mean value recorded. For the nonparametric data, 1 score point difference at most was recorded with respect to intrasubject examination of the 5 pictures. Intraobserver and interobserver variability showed values of the same magnitude, both for parametric and nonparametric data.

The caliber of visible capillary loops was $15.69 \pm 2.07$ $\mu \mathrm{m}$ in the nonsmokers group and $11.66 \pm 1.62 \mu \mathrm{m}$ in the smokers group $(P<.001)$. The number of visible capillary loops was $4.47 \pm 0.98$ in the nonsmokers group and $6 \pm 1.41$ in the smokers group $(P<.001)$. The background optical transmittance was $192.07 \pm$ 7.07 in the nonsmokers group and $166.75 \pm 5.32$ in the smokers group $(P<.001)$. All the previously mentioned data are expressed as mean $\pm \mathrm{SD}$.

Tortuousness of capillary loops score ranged from 0 to 1 (median, 1) in the nonsmokers group and from 1 to 3 (median, 2$)$ in the smokers group $(P<.001)$. In the nonsmokers group, microaneurysms and microhemorrhages were absent. In the smokers group, 15 subjects (30\% of the group) had microaneurysms alone, and 17 subjects (34\% of the group) had both microaneurysms and microhemorrhages. In no cases were microhemorrhages present without microaneurysms. The total score (tortuousness + microaneurysms + microhemorrhages) ranged from 1 to 5 (median, 3) in the smokers group and from 0 to 1 (median, 1$)$ in the nonsmokers group $(P<.001)$.

Figure 4 shows an example of normal videocapillaroscopic pattern, with its lighter and rose-shaded background, and Figure 5 shows an example of heavy 


\section{Figure 1}
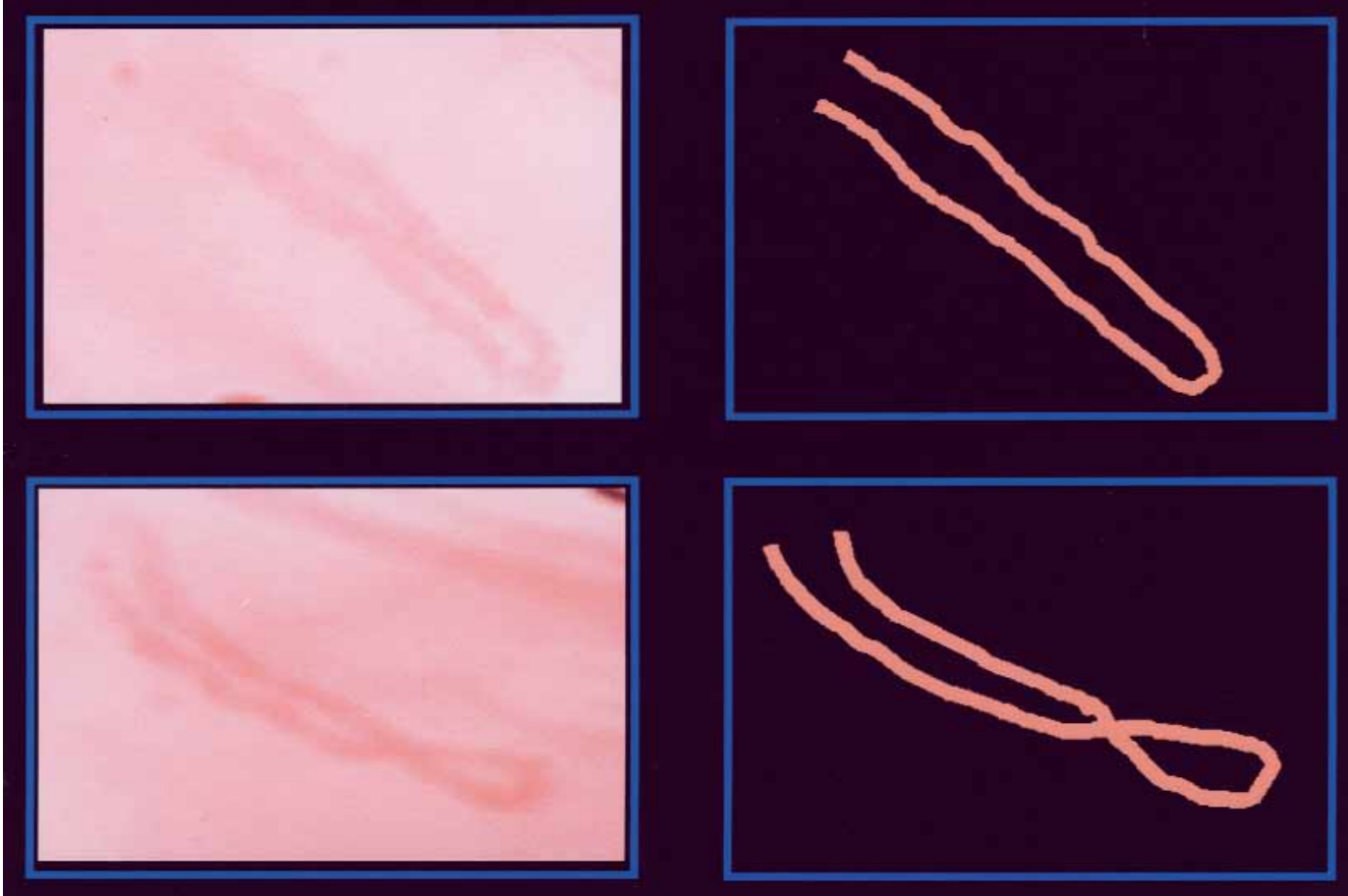

Top, Tortuousness score 0 (no tortuousness or "hairpin" aspect of capillary loop). Bottom, Tortuousness score 1 (single twist tortuousness of capillary loop).

Figure 2

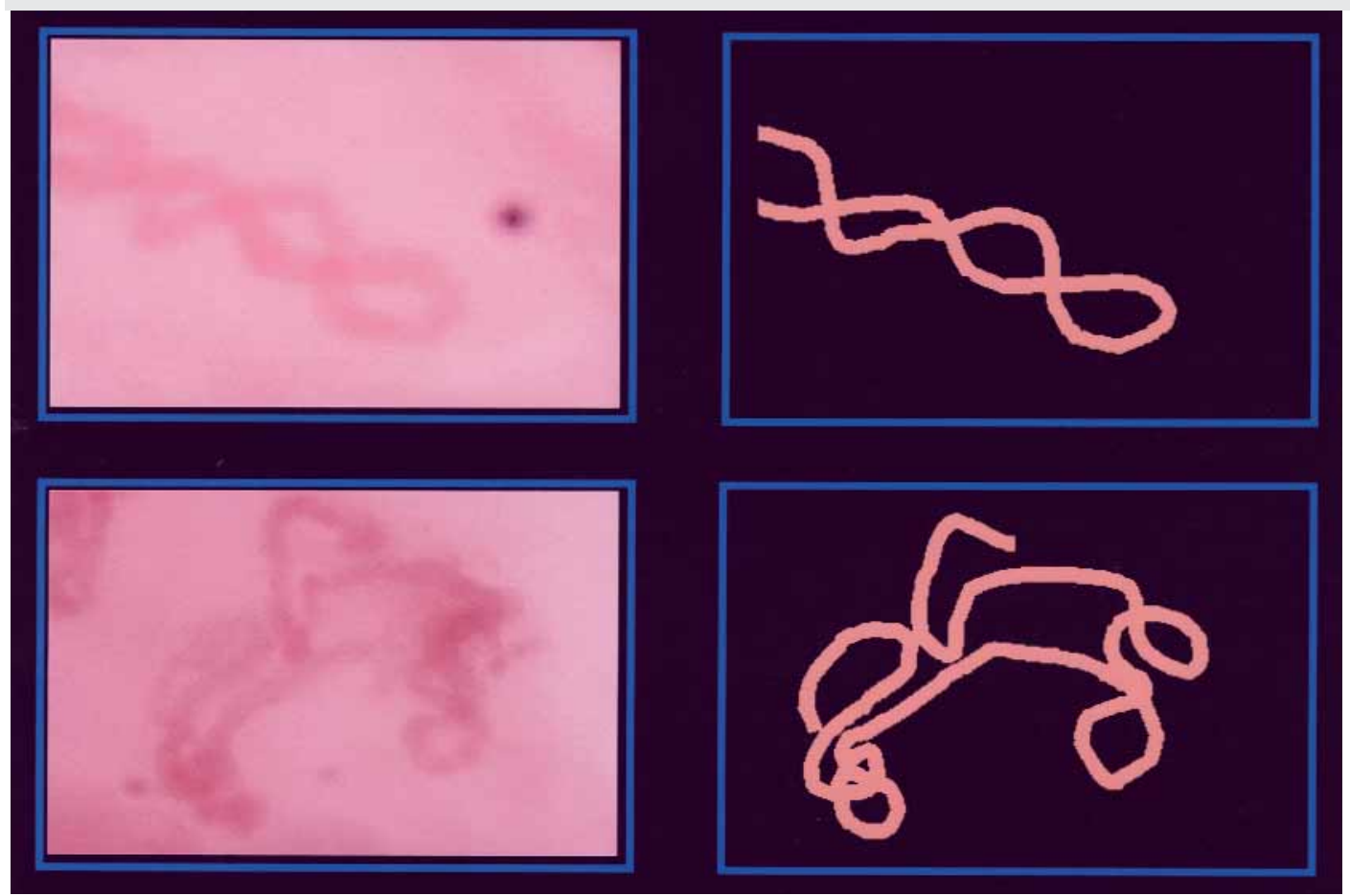

Top, Tortuousness score 2 (multiple twist tortuousness or "cork-screw" aspect of capillary loop). Bottom, Tortuousness score 3 (complete distortion of capillary loop or "ball" aspect). 


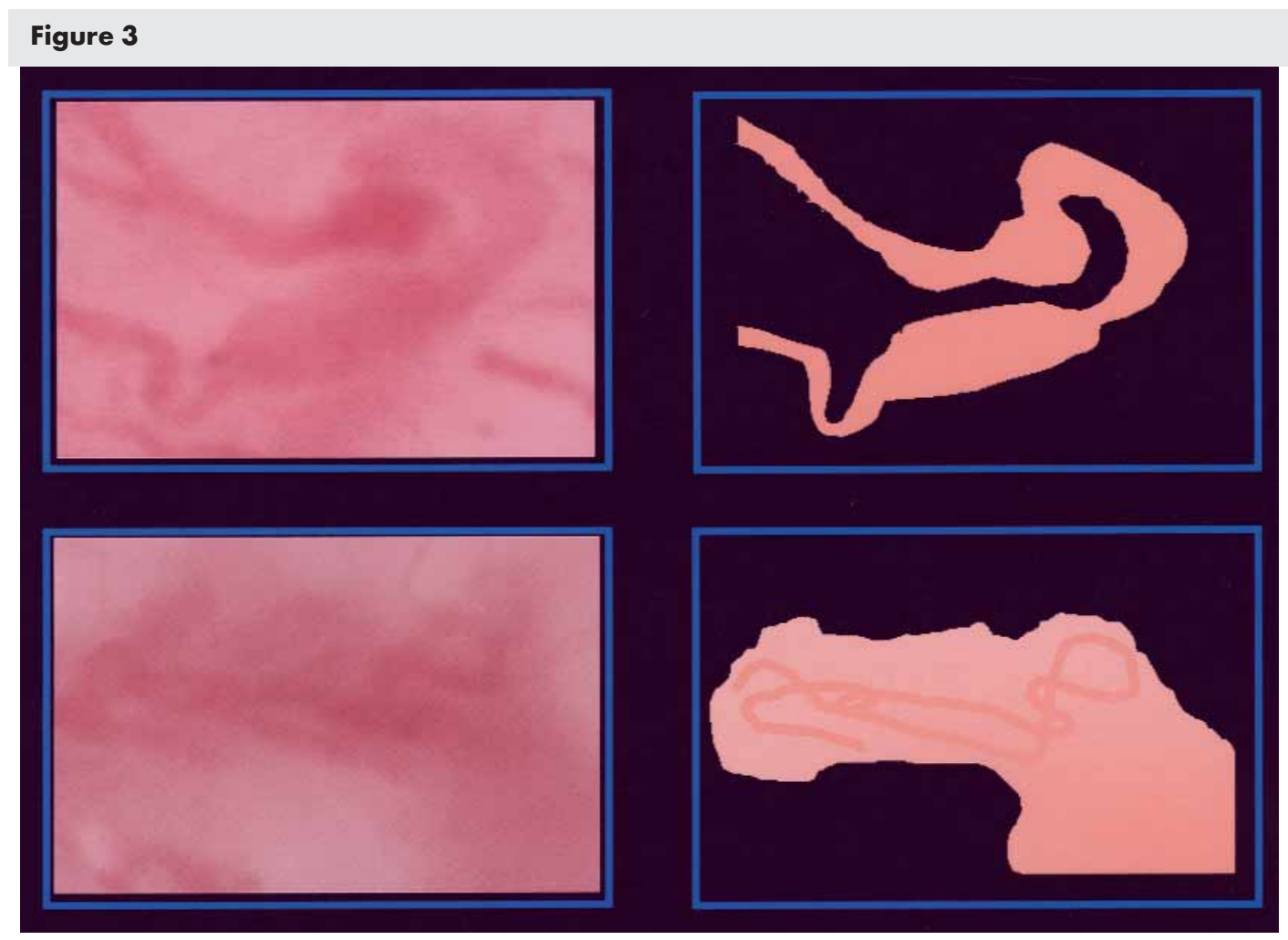

Top, Example of microaneurysm. Bottom, Example of microhemorrhagic stain, with small and completely distorted capillary loop inside.

smoker videocapillaroscopic pattern, with its darker and violet-shaded background.

No significant correlation was found between the parametric data and cumulative smoking habit $(P=$ not significant). A significant correlation was found between cumulative smoke and tortuousness of capillary loops $(P<.001)$ and between cumulative smoke and total score $(P<.005)$.

\section{Discussion}

Our data show that chronic smoking habit does induce significant morphologic changes in the microcirculation of the human labial mucosa and that some of these changes are strongly related to cumulative smoking habit. To our knowledge, no evidence exists on morphologic changes induced by smoke in the microcirculation. Our discussion will focus on the question of whether our original morphologic data fit previous functional and experimental findings and on the implications of our study.

The decreased caliber of capillary loops, found in the smokers group, confirms previous findings. In fact, it has been shown that acute cigarette smoking significantly impairs human cutaneous ${ }^{1}$ and coronary ${ }^{6}$ microcirculation and that chronic tobacco smoke increases postcapillary venous pressure but not precapillary arterial pressure in the mesenteric microcirculation of the rat, ${ }^{7}$ thus resulting in a decreased capillary flow caused by a reduced pressure gradient across the capillaries. Furthermore, although the effects of acute cigarette smoking are not related to the nicotine content of the cigarettes and cannot be obtained with the acute administration of transdermal nicotine, 8 nicotine seems to perform important interactions with endogenous substances (norepinephrine, acetylcholyne, adenosine diphosphate [ADP]). It has been shown, in the hamster cheek pouch, that nicotine potentiates norepinephrine-induced vasoconstriction of arterioles $^{2}$ and that both nicotine and cigarette smoke extract profoundly and selectively impair endothelium-dependent vasodilatation of arterioles. ${ }^{9}$

The increased number of visible capillary loops, found in the smokers group of our study, is a newly recorded finding; no previous data regarding smoke and microcirculation interactions are available in the literature with special reference to that. We suggest that the increase in the number of visible capillary loops could be the result of a recruitment of nonperfuse capillary loops caused by the venoarteriolar reflex, as 


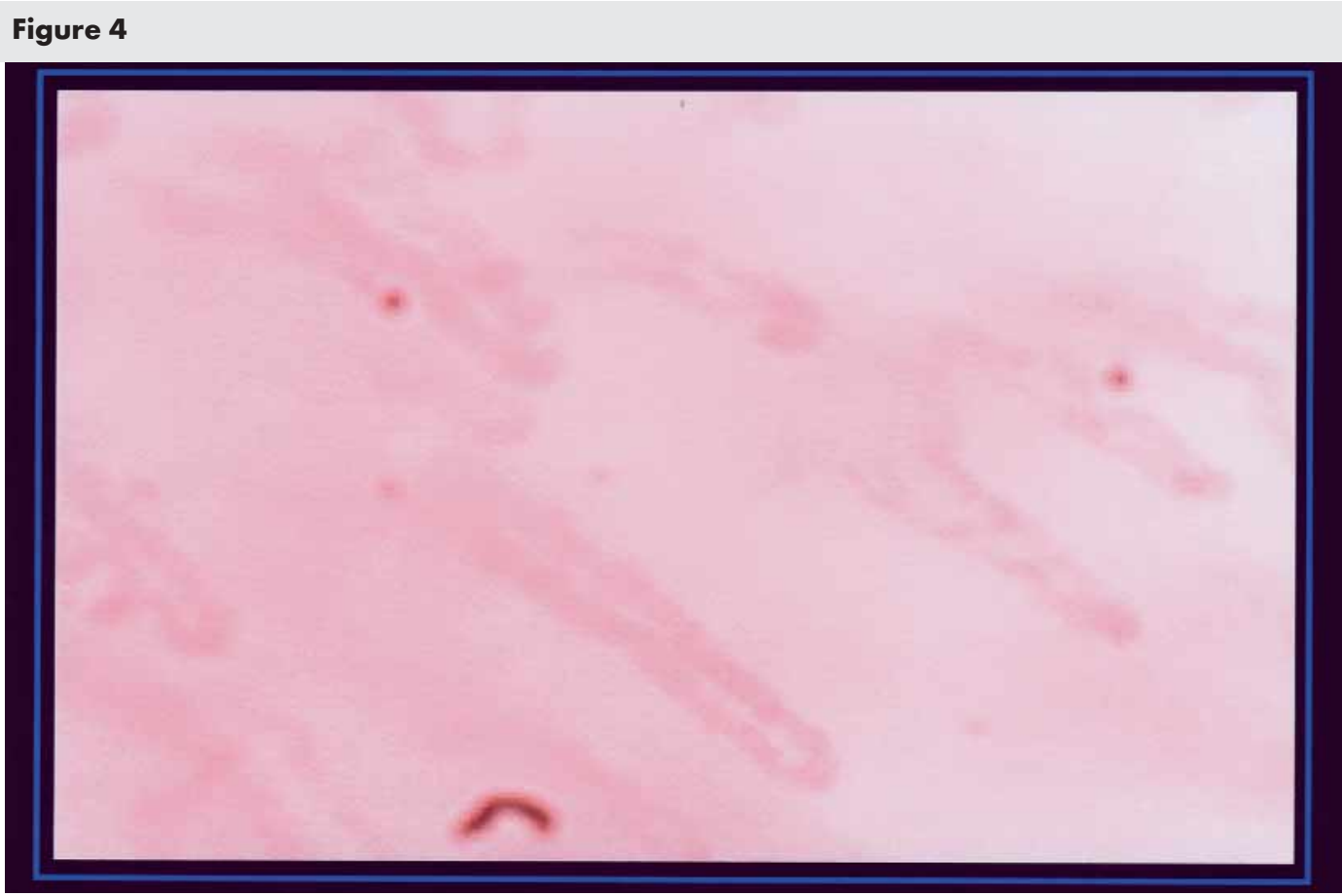

Example of normal videocapillaroscopic pattern: "hairpin" aspect of capillary loops on lighter and rose-shaded background.

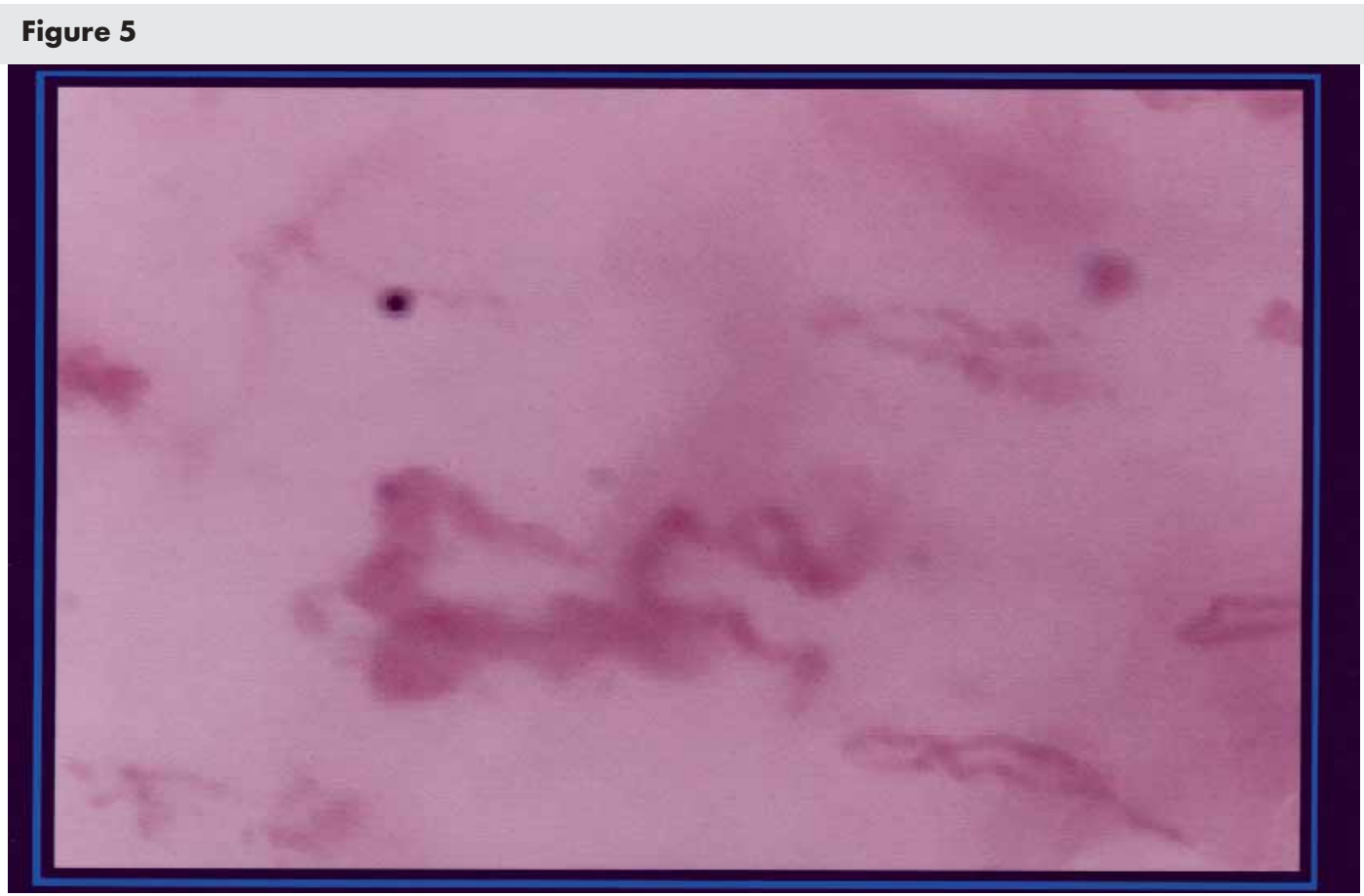

Example of heavy smoker videocapillaroscopic pattern: aneurysmatic distorted capillary loop and some hemorrhagic stain on darker and violet-shaded background. 
shown in chronic venous insufficiency ${ }^{10}$ and in critical limb ischemia, ${ }^{11}$ triggered by the smoke-induced increase in postcapillary venular pressure. ${ }^{7}$

The reduced background optical transmittance, found in the smokers group, can be considered the expression of the venular congestion, and the background color, with its violet shade (see Figure 5), suggests this hypothesis. Venular congestion would be related to the increase in postcapillary venous pressure induced by chronic tobacco smoke ${ }^{7}$ and to the fact that both nicotine and cigarette smoke extract, which cause a significant increase in leukocyte aggregation and adhesion in the cerebral microcirculation of the mouse ${ }^{3}$ and in hamster dorsal skin fold chamber, ${ }^{12}$ exert this effect primarily, although not exclusively, at the postcapillary endothelial surface. ${ }^{12}$ Furthermore, the decreased human leukocytes deformability, shown after acute cigarette smoking, ${ }^{4}$ and the increase of human red blood cells volume, transit time, and number and size of red blood cells aggregates, shown in chronic smokers, ${ }^{5}$ could play an important additional role in causing venular congestion.

With regard to the increased tortuousness of capillary loops, the score 1 pattern or single-twist pattern (Figure 1) is likely the result of the nonperfect parallelism between the plane of the capillary loop and the plane of the camera and hence should not be considered a pathologic pattern. Indeed, the score 1 pattern was largely represented even in the nonsmokers group (90\%). The tortuousness of capillary loops is a common capillaroscopic finding in systemic diseases, such as diabetes and rheumatic diseases, but the underlying pathomechanism is largely unknown. With regard to the increased tortuousness of capillary loops found in smokers, it has been shown that cigarette smoke causes abnormal pattern formation of chick chorioallantoic membrane blood vessels and alters the composition of the extra cellular matrix in the chorioallantoic membrane mesoderm ${ }^{13}$ and that nicotine stimulates the proliferation in vascular endothelial bovine cells. ${ }^{14}$ We suggest that the increased tortuousness of the capillary loops could be related to the neoangiogenetic effect of nicotine.

Microaneurysms and microhemorrhages are common capillaroscopic findings in systemic diseases, such as diabetes, rheumatic diseases, and hypertension. With regard to the presence of microaneurysms, alone or associated with microhemorrhages, in the smokers group, this finding could be reasonably attributed to the postcapillary venular congestion. Furthermore, it has been shown that cigarette smoke extract per se does not alter basal microvascular permeability but potentiates bradykinin-induced increase in microvascular permeability. ${ }^{15}$

Why there is no correlation between the cumulative smoking habit and the parametric data, although there is a strong correlation between the cumulative smoke and the nonparametric data, is unclear. We suggest that different kinetics of onset of the different types of damages could correctly address the question. The decreased caliber of capillary loops, the increased number of visible capillary loops, and the reduced background optical transmittance could be early effects of smoke because of their predominant functional aspect and hence not related to the cumulative smoke. Tortuousness of capillary loops, presence of microaneurysms and of microhemorrhages, on the contrary, could be late damages because of their predominant structural aspect and hence actually related to the cumulative smoking habit.

In conclusion, our morphologic data, as a whole, fit previously reported functional and experimental findings. Our study confirms that videocapillaroscopy is a simple, safe, sensitive, reliable, and low-cost technique, comfortable for the patient and easy to perform for the operator. Furthermore, our study shows that videocapillaroscopy, besides being used to investigate the microcirculation in the context of diseases already known to affect the microvessels, such as rheumatic diseases or diabetes, can also be used to investigate the microvascular damages in any disease or clinical condition, offering new insight into the pathologic mechanisms of tissue damage. Our original findings indicate that chronic smoking habit does induce significant morphologic changes in the microcirculation of the human labial mucosa and that some of these changes are strongly related to cumulative smoke.

\section{References}

1. Zhang J, Ying $X$, Lu $Q$, et al. A single dose of vitamin $C$ counteracts the acute negative effect on microcirculation induced by smoking a cigarette. Microvasc Res 1999;58:305-11.

2. Mayhan WG. Acute infusion of nicotine potentiates norepinephrineinduced vasoconstriction in the hamster cheek pouch. J Lab Clin Med 1999; 133:48-54.

3. Yong $T$, Zheng $M Q$, Linthicum DS. Nicotine induces leukocyte rolling and adhesion in the cerebral microcirculation of the mouse. J Neuroimmunol 1997;80:158-64.

4. Drost EM, Selby C, Bridgeman MM, et al. Decreased leukocyte deformability after acute cigarette smoking in humans. Am Rev Respir Dis 1993; 148:1277-83.

5. Maurel A, Apovo M, Beuzard Y, et al. Effects of smoking on blood rheology. J Mal Vasc 1997;22:239-43.

6. Kaufman PA, Gnecchi-Ruscone T, Terlizzi M, et al. Coronary heart disease in smokers: vitamin $C$ restores coronary microcirculatory function. Circulation 2000;102:1233-8.

7. Richardson D, Coates F, Morton R. Early effects of tobacco smoke exposure on vascular dynamics in the microcirculation. J Appl Physiol 1975;39:119-23

8. Fulcher SM, Koman LA, Smith BP, et al. The effect of transdermal nicotine on digital perfusion in reformed habitual smokers. J Hand Surg 1998;23:792-9.

9. Mayhan WG, Patel KP. Effect of nicotine on endothelium-dependent arteriolar dilatation in vivo. Am J Physiol 1997;272:H2337-42. 
10. Svedman C, Cherry GW, Ryan TJ. The veno-arteriolar reflex in venous leg ulcer patients studied by Laser-Doppler imaging. Acta Derm Venereol 1998;78:258-61.

11. Khiabani HZ, Anvar MD, Kroese AJ. The role of the veno-arteriolar reflex in the pathogenesis of peripheral oedema in patients with chronic critical limb ischaemia. Ann Chir Gynaecol 2000;89:93-8.

12. Lehr HA, Frei B, Arfos KE. Vitamin $C$ prevents cigarette smokeinduced leukocyte aggregation and adhesion to endothelium in vivo. Proc Natl Acad Sci U S A 1994;91:7688-92.
13. Melkonian G, Le C, Zheng W, et al. Normal patterns of angiogenesis and extracellular matrix deposition in chick chorioallantoic membranes are disrupted by mainstream and sidestream cigarette smoking. Toxicol Appl Pharmacol 2000;163:26-37.

14. Villablanca AC. Nicotine stimulates DNA synthesis and proliferation in vascular endothelial cells in vitro. J Appl Physiol 1998;84:208998.

15. Mayhan WG, Rubinstein I. Cigarette smoke extract potentiates bradykinin-induced increases in microvascular permeability. J Appl Physiol 1993;75:27-32. 